\title{
Pacific
}

Journal of

Mathematics

\section{WITT RINGS UNDER ODD DEGREE EXTENSIONS}

ROBERT FITZGERALD 


\title{
WITT RINGS UNDER ODD DEGREE EXTENSIONS
}

\author{
ROBERT W. FitzGERALD
}

\begin{abstract}
For a separable odd degree field extension $K / F$ the kernel of a Scharlau transfer of Witt rings $s_{*}: W K \rightarrow W F$ is a $W F$-module. We compute the prime ideals attached to ker $s_{*}$ and deduce that $W K$ is not a projective $W F$-module if an ordering on $F$ extends uniquely to $K$. An example shows $W K$ may be a free $W F$-module if $F$ is real and no ordering extends uniquely. For non-real, non-rigid $F$ we show that $K / F$ Galois and $W K$ noetherian implies $W K$ is not a projective $W F$-module.
\end{abstract}

If $K / F$ is a finite extension of fields (characteristic not 2) then each non-trivial linear functional $s: K \rightarrow F$ induces a Scharlau transfer $s_{*}: W K \rightarrow W F$ on the Witt rings. When $K=F(\sqrt{d})$ the kernel and image of $s_{*}$ are well known. We restrict our attention to separable odd degree extensions, where $s_{*}$ is surjective but little is known of ker $s_{*}$. The map induced by inclusion $r_{*}: W F \rightarrow W K$ is injective and we view $W F$ as a subring of $W K$. Then $W K$ and ker $s_{*}$ are $W F$-modules and our approach is module theoretic.

$W F$ need not be noetherian and ker $s_{*}$ need not be finitely generated over $W F$. So the usual theory of prime ideals associated to modules must be replaced by the notion of attached primes (in the sense of Dutton). We show no $P(\alpha, p)$ is attached to ker $s_{*}, P(\alpha)$ is attached iff $\alpha$ has more than one extension to $K$ and $I F$ is attached iff $W_{t} K \cap$ ker $s_{*} \neq 0$. As a consequence, $W K=W F$ iff each ordering on $F$ extends uniquely to $K$ and $W_{t} K \cap \operatorname{ker} s_{*}=0$. Another consequence is that $W K$ is finitely generated over $W F$ if $F$ has only finitely many orderings and $I F$ is not attached to ker $s_{*}$.

The main result deduced from the work on attached primes is that $W K$ is not a projective $W F$-module if some ordering on $F$ extends uniquely to $K$. WK may be projective, however, if $F$ is real and no ordering extends uniquely. We present an example where $K / F$ is Galois, $F$ is real, both $W K$ and $W F$ noetherian rings and $W K$ is a free $W F$-module. When $F$ is non-real and non-rigid we show the same conditions $(K / F$ Galois, $W K$ and $W F$ noetherian) implies $W K$ is not a free $W F$-module. Weaker results hold under fewer restrictions on $K / F$. 
The first section gives basic results and several examples. The last section concerns the possible values of $[G(K): G(F)]$ when this is finite (here $G(E)=E^{*} / E^{\cdot 2}$ ). Two sample results: If $K / F$ is Galois and $[K: F]=p$ a prime then $p$ divides $[G(K): G(F)]-1$. If $K / F$ has a real normal closure then $[K: F] \leq[G(K): G(F)]$.

$\operatorname{Hom}(K, F)^{\cdot}$ denotes the non-trivial linear functionals $s: K \rightarrow F$. The set of orderings on a field $E$ is denoted $X_{E}$. If $\alpha \in X_{F}$ then $X(\alpha)=\left\{\beta \in X_{K}|\beta| F=\alpha\right\}$. For $\alpha \in X_{F}$ and an odd prime $p$ we write $P(\alpha, p)$ for $\left\{r \in W F \mid \operatorname{sgn}_{\alpha} r \equiv 0(\bmod p)\right\}$ and $P(\alpha)=\{r \in$ $\left.W F \mid \operatorname{sgn}_{\alpha} r=0\right\}$. These ideals, with $I F=\{r \in W F \mid \operatorname{dim} r \equiv 0$ $(\bmod 2)\}$, are the prime ideals of $W F$.

$W_{t} F$ denotes the torsion part of $W F$. The height of $F, h(F)$, is the least positive $k$ such that $2^{k} \cdot W_{t} F=0$ (or infinity if no such $k$ exists). If $R_{1}$ and $R_{2}$ are Witt rings then the fiber product $R_{1} \sqcap R_{2}=$ $\left\{\left(r_{1}, r_{2}\right) \mid r_{i} \in R_{i}, \operatorname{dim} r_{1} \equiv \operatorname{dim} r_{2}(\bmod 2)\right\}$ is again a Witt ring. If $C$ is a group of exponent two then the group ring $R_{1}[C]$ is again a Witt ring.

\section{Basic facts.}

Definition. (i) $m(K / F)=\bigcap \operatorname{ker} s_{*}$, over all $s \in \operatorname{Hom}(K, F)^{\circ}$.

(ii) $M(K / F)=\sum \operatorname{ker} s_{*}$, over all $s \in \operatorname{Hom}(K, F)^{\circ}$.

Lemma 1.1. Let $s \in \operatorname{Hom}(K, F)^{\cdot}$.

(1) $\operatorname{ker} s_{*}$ is a $W F$-submodule of $W K$.

(2) If $t \in \operatorname{Hom}(K, F)^{\cdot}$ then $\operatorname{ker} s_{*}=\langle z\rangle \operatorname{ker} t_{*}$ for some $z \in K^{\cdot}$.

(3) $m(K / F)=\left[\operatorname{ker} s_{*}: W K\right]$ is an ideal of $W K$.

(4) $M(K / F)$ is the ideal generated by $\operatorname{ker} s_{*}$.

(5) There exists $t \in \operatorname{Hom}(K, F)^{\cdot}$ with $t_{*}\langle 1\rangle=\langle 1\rangle$.

(6) If $s_{*}\langle 1\rangle=\langle 1\rangle$ then $W K \approx W F \oplus \operatorname{ker} s_{*}$.

(7) If $s_{*}\langle 1\rangle=\langle 1\rangle$ then $\operatorname{ker} s_{*}$ is generated (over $W F$ ) by $\{\langle x\rangle-$ $\left.s_{*}\langle x\rangle \mid x \in K^{\bullet}\right\}$.

Proof. (1) $s_{*}$ is additive and if $\phi \in \operatorname{ker} s_{*}$ and $r \in R$ then $s_{*}(r \phi)$ $=r s_{*}(\phi)=0$. Thus ker $s_{*}$ is a $W F$-submodule of $W K$.

(2) There exists $z \in K^{\cdot}$ such that $s(x)=t(z x)$ for all $x \in K$. Then $s_{*}(\phi)=t_{*}(\langle z\rangle \phi)$ for all $\phi \in W K$ and so ker $s_{*}=\langle z\rangle$ ker $t_{*}$.

(3) Let $\phi \in m(K / F)$ and $z \in K^{\circ}$. Define $t(x)$ to be $s(z x)$ for all $x \in K$. Then $\phi \in \operatorname{ker} t_{*}=\langle z\rangle \operatorname{ker} s_{*}$. Since $z$ was arbitrary, we have $\phi \in\left[\operatorname{ker} s_{*}: W K\right]$. Conversely, if $\phi \in\left[\operatorname{ker} s_{*}: W K\right]$ then for every $z \in K^{\cdot},\langle z\rangle \phi \in \operatorname{ker} s_{*}$ and $\phi \in\langle z\rangle \operatorname{ker} s_{*}=\operatorname{ker} t_{*}$, for some 
$t \in \operatorname{Hom}(K / F)^{\circ}$. Thus $\phi \in m(K / F)$. Clearly [ker $\left.s_{*}: W K\right]$ is an ideal.

(4) $M(K / F)=\sum \operatorname{ker} t_{*}=\sum\langle z\rangle \operatorname{ker} s_{*}$ is the ideal generated by $\operatorname{ker} s_{*}$.

(5) We may write $K=F(x)$ since $K$ is separable over $F$. Take $t \in \operatorname{Hom}(K, F)^{\circ}$ with $t(1)=1$ and $t(x)=\cdots=t\left(x^{n-1}\right)=0 \quad(n=$ $[K: F])$. Then $t_{*}\langle 1\rangle=\langle 1\rangle$ by $[15$, II 5.8$]$.

(6) If $s_{*}\langle 1\rangle=\langle 1\rangle$ then the exact sequence $0 \rightarrow \operatorname{ker} s_{*} \rightarrow W K \rightarrow$ $W F \rightarrow 0$ splits. This also proves (7).

There are few examples of Witt rings under odd degree extensions in the literature. We present several to illustrate the range of possible $m(K / F)$ and $M(K / F)$.

ExAmples. (1) The definitions of $m(K / F)$ and $M(K / F)$ make sense for any finite extension $F \subset K$. Consider $K=F(\sqrt{d})$ and define $s: K \rightarrow F$ by $s(1)=0, s(\sqrt{d})=1$. Then ker $s_{*}=r_{*}(W F)$. Since $\langle 1\rangle \in \operatorname{ker} s_{*}$ we have $M(K / F)=W K$. Also, $m(K / F)=$ $\operatorname{ann}_{W F}\left(\operatorname{ann}_{W F}\langle 1,-d\rangle\right) \otimes K$ by $[5,2.12]$. Note that if $W F$ is Gorenstein (e.g., a group ring extension of a Witt ring of local type) then $\operatorname{ann}_{W F}\left(\operatorname{ann}_{W F}\langle 1,-d\rangle\right)=(\langle 1,-d\rangle)$ and hence $m(K / F)=0$ (cf. [9]).

(2) Let $F=\mathbf{Q}_{2}$ and $K=\mathbf{Q}_{2}(e)$ where $e$ is a root of $x^{3}+$ 2. Then $K^{\cdot} / K^{\cdot 2}$ may be represented by the group generated by $\langle 2\rangle,\langle 3\rangle,\langle 5\rangle,\langle\alpha\rangle,\langle\beta\rangle$ where $\alpha=2+e^{2}$ and $\beta=1+e^{2}$. Define $s: K \rightarrow F$ by $s(1)=1, s(e)=0$ and $s\left(e^{2}\right)=0$. Then $s_{*}\langle 1\rangle=$ $\langle 1\rangle, s_{*}\langle\alpha\rangle=\langle 3\rangle, s_{*}\langle\beta\rangle=\langle 5\rangle$ and $s_{*}\langle\alpha \beta\rangle=\langle 2\rangle\langle 1,-7,-14\rangle \simeq$ $\langle 2\rangle\langle 1,1,2\rangle \simeq\langle 1,1,1\rangle$ (see [15, p. 188]). Set $\rho=4 \cdot\langle 1\rangle$ and $\chi=3 \cdot\langle 1\rangle$.

We verify that $m(K / F)=0$. Let $\phi=r_{1}+r_{2}\langle\alpha\rangle+r_{3}\langle\beta\rangle+r_{4}\langle\alpha \beta\rangle \epsilon$ $m(K / F)$ with $r_{i} \in W F$. From $s_{*} \phi=0, s_{*}\langle\alpha\rangle \phi=0$ and $s_{*}\langle\beta\rangle \phi=0$ we obtain:

$$
\begin{aligned}
r_{1}+\langle 3\rangle r_{2}+\langle 5\rangle r_{3}+\chi r_{4} & =0, \\
\rho r_{3}+\rho r_{4} & =0, \\
\rho r_{2}+\rho r_{4} & =0 .
\end{aligned}
$$

The last two equations imply $\operatorname{dim} r_{2} \equiv \operatorname{dim} r_{3} \equiv \operatorname{dim} r_{4}(\bmod 2)$. The first equation yields $\phi=\langle\alpha,-3\rangle r_{2}+\langle\beta,-5\rangle r_{3}+(\langle\alpha \beta\rangle-\chi) r_{4}$. When all $r_{i}(2 \leq i \leq 4)$ are even dimensional then $\phi \in I^{2} K$. When all $r_{i}$ are odd dimensional then $d(\phi)=1$ and again $\phi \in I^{2} K$. But $I^{2} K=\{0, \rho\}$ and $s_{*}(\rho)=\rho \neq 0$. Thus $\phi=0$.

Lastly, $M(K / F)=(\langle 1,-3 \alpha\rangle,\langle 1,-5 \beta\rangle)$. Namely, $M(K / F)$ is 
generated by $\langle 1,-3 \alpha\rangle,\langle 1,-5 \beta\rangle$ and $\chi-\langle\alpha \beta\rangle$. Now $\rho \in\langle 1,-3 \alpha\rangle I K$ and $\chi-\langle\alpha \beta\rangle=\rho-\langle 1, \alpha \beta\rangle=\rho-\langle 15\rangle(\langle 1,-3 \alpha\rangle+\langle 3 \alpha\rangle\langle 1,-5 \beta\rangle)$.

(3) Let $F=\mathbf{C}(x)$. It is easy to see $t^{3}+x t+x$ is irreducible over $F$. Let $\alpha$ be a root and let $K=F(\alpha)$. Pick $s \in \operatorname{Hom}(K, F)^{\circ}$ with $s_{*}\langle 1\rangle=\langle 1\rangle$. Now for all $u \in K, s_{*}\langle u\rangle=\left\langle N_{K / F}(u)\right\rangle+\phi$, for some $\phi \in$ $I^{2} K=0$. We are using here that $K$ is a $C_{1}$-field for every finite extension [15, II 15.2]. So $s_{*}\langle u\rangle=\left\langle N_{K / F}(u)\right\rangle$, and $s_{*}$ is a ring homomorphism. Thus $m(K / F)=\operatorname{ker} s_{*}=M(K / F)=\left\{\langle 1,-u\rangle \mid N_{K / F}(u)=1\right\}$.

This is the only example (of the three) for which $m(K / F) \neq 0$. To verify this it is enough to show $-x \alpha \notin K^{2}$ as $N_{K / F}(-x \alpha) \in F^{2}$. But if $-x \alpha=\left(a+b \alpha+c \alpha^{2}\right)^{2}$ then $b=a^{2} / 2 c x$ and $(a / c)^{4}+8(a / c) x^{2}=4 x^{3}$. However $t^{4}+8 x^{2} t-4 x^{3}$ has no roots in $F$.

(4) In $\S 3$ an extension $F \subset K$ will be constructed with $W F \approx \mathbf{Z}$ and $W K \approx \mathbf{Z}^{3}$. Here $\dot{F} / \dot{F}^{2}=\{ \pm 1\}$ and $\dot{K} / \dot{K}^{2}=\{ \pm 1, \pm \alpha, \pm \beta$, $\pm \alpha \beta\}$. Here $\alpha$ corresponds to $(1,-1,-1) \in \mathbf{Z}^{3}$ and $\beta$ corresponds to $(-1,1,-1)$. There is, by a later result $(1.4)$, an $s \in \operatorname{Hom}(K, F)^{\circ}$ with $s_{*}\langle 1\rangle=\langle 1\rangle, s_{*}\langle\alpha\rangle=\langle 1\rangle, s_{*}\langle\beta\rangle=\langle 1\rangle$ and $s_{*}\langle\alpha \beta\rangle=-3\langle 1\rangle$. Thus ker $s_{*}$ is generated by $\langle 1,-\alpha\rangle,\langle 1,-\beta\rangle,\langle 1,1,1, \alpha \beta\rangle$. Using $\langle 1, \alpha, \beta, \alpha \beta\rangle=0$ it is straightforward to show $m(K / F)=0$ and $M(K / F)=(\langle 1,-\alpha\rangle,\langle 1,-\beta\rangle)$.

For any field $E$ let $G(E)=E^{\cdot} / E^{\cdot 2}$. Set $U=\left\{\langle x\rangle \in G(K) \mid N_{K / F}(x)\right.$ $\left.\in \dot{F}^{2}\right\}$.

LEMMA 1.2. $G(K) \approx U \times G(F)$.

Proof. The sequence $1 \rightarrow U \rightarrow G(K) \rightarrow G(F) \rightarrow 1$ is exact and splits since for $a \in F^{*}$ we have $N_{K / F}(a)=a^{m}$ where $m=[K: F]$ is odd and so $N_{K / F}(a) \in a \dot{F}^{2}$.

LEMMA 1.3. If $s_{*}\langle 1\rangle=\langle 1\rangle$ and $\operatorname{dim}\left(s_{*}\langle x\rangle\right)_{a n}=1$ for some $x \in K^{*}$ then $s_{*}\langle x\rangle=\left\langle N_{K / F}(x)\right\rangle$.

Proof. Suppose $[K: F]=2 k+1$. Then $s_{*}\langle 1\rangle \simeq k \cdot\langle 1,-1\rangle+\langle 1\rangle$ so that $\operatorname{det}\left(s_{*}\langle 1\rangle\right)=(-1)^{k}$. Hence $\operatorname{det}\left(s_{*}\langle x\rangle\right)=(-1)^{k} N_{K / F}(x)[15$, II 5.12] and so $s_{*}\langle x\rangle=\left\langle N_{K / F}(x)\right\rangle$.

Proposition 1.4. Let $s \in \operatorname{Hom}(K, F)^{\cdot}$ with $s_{*}\langle 1\rangle=\langle 1\rangle$. Set $L(s)=\left\{\langle y\rangle \in G(K) \mid N_{K / F}(y) \in F^{2}\right.$ and $\left.s_{*}\langle y\rangle=\langle 1\rangle\right\}$. Then:

(1) $\{\langle 1,-y\rangle \mid y \in L(s)\} \subset$ ker $s_{*}$, and

(2) $L(s) L(s)=U$. 
Proof. (1) is clear as is the inclusion $L(s) L(s) \subset U$. Suppose then that $\beta \in U$ and set $E=F(\beta)$. Define $v: E \rightarrow F$ by $v(1)=1$ and $v\left(\beta^{i}\right)=0,1 \leq i<[E: F]$. Then $v_{*}\langle 1\rangle=\langle 1\rangle$ and $v_{*}\langle\beta\rangle=\langle 1\rangle$ [15, II 5.8] (note $N_{E / F}(\beta)=1$ as $1=N_{K / F}(\beta)=N_{E / F}\left(N_{K / E}(\beta)\right)=$ $N_{E / F}(\beta)$, modulo squares). Pick any $u \in \operatorname{Hom}(K, E)^{\circ}$ with $u_{*}\langle 1\rangle=$ $\langle 1\rangle$. Then $(v u)_{*}\langle 1\rangle=\langle 1\rangle$ and $(v u)_{*}\langle\beta\rangle=v_{*}\left(u_{*}\langle\beta\rangle\right)=v_{*}\langle\beta\rangle=\langle 1\rangle$, as $\beta \in E$. Thus $\{1, \beta\} \subset L(v u)$. Now there exists $z \in K^{*}$ with $v u(x)=s(z x)$ for all $x \in K$. Note $\langle 1\rangle=(v u)_{*}\langle 1\rangle=s_{*}\langle z\rangle$ so that $N_{K / F}(z) \in F^{2}$ by (1.3). Also $z L(v u)=L(s)$. Thus $z, z \beta \in L(s)$ and $\beta \in L(s) L(s)$.

Proposition 1.5. $m(K / F) \subset W_{t} K$, the torsion ideal of $W K$.

Proof. If $x \in K^{\cdot}$ and $\phi \in m(K / F)$ then $\operatorname{tr}_{*}(\langle x\rangle \phi)=0$ where tr is the trace map $\operatorname{tr}_{K / F}$. Let $Q \in X_{K}$ and let $P=Q \cap F$. Since $X(P)$ is finite, we may find a Pfister form $p$ and integer $m$ with $\operatorname{sgn}_{Q}(p)=2^{m}$ and $\operatorname{sgn}_{Q^{\prime}}(p)=0$ for $Q^{\prime} \in X(P)-\{Q\}$. Then by [15, III 4.5]:

$$
0=\operatorname{sgn}_{p} \operatorname{tr}_{*}(p \phi)=\sum_{Q^{\prime} \in X(P)} \operatorname{sgn}_{Q^{\prime}}(p \phi)=2^{m} \operatorname{sgn}_{Q}(\phi) .
$$

Thus $\operatorname{sgn}_{Q}(\phi)=0$ and as $Q$ was arbitrary, we have $\phi \in W_{t} K$.

Proposition 1.6. Suppose $s \in \operatorname{Hom}(K, F)^{\bullet}$ satisfies $s_{*}\langle 1\rangle=\langle 1\rangle$. Let $m=[K: F]$ and set $k=(m-1) / 2$ and $n=m-(-1)^{k}$. Let $J \subset W K$ be the ideal generated by $\{\langle 1,-y\rangle \mid y \in U\}$. Then:

(1) $M(K / F)=J+\left(\left\{\langle 1\rangle-s_{*}\langle y\rangle \mid y \in U\right\}\right)$.

(2) If $K / F$ is Galois then $n \cdot\langle 1\rangle \in M(K / F)$.

(3) If $K / F$ is Galois then $M(K / F)=J$.

Proof. (1) $J \subset M(K / F)$ by (1.4). If $N_{K / F}(y) \in F^{2}$ then $\langle y\rangle-$ $s_{*}\langle y\rangle \in \operatorname{ker} s_{*} \subset M(K / F)$ and $\langle 1\rangle-s_{*}\langle y\rangle=\langle 1,-y\rangle+\langle y\rangle-s_{*}\langle y\rangle \in$ $M(K / F)$. Conversely, $M(K / F)$ is generated by ker $s_{*}$, by (1.1), which is generated by $\langle y\rangle+s_{*}\langle y\rangle$, for $y \in U$. And $\langle y\rangle-s_{*}\langle y\rangle=$ $-\langle 1,-y\rangle+\left(\langle 1\rangle-s_{*}\langle y\rangle\right) \in J+\left(\left\{\langle 1\rangle-s_{*}\langle y\rangle \mid y \in U\right\}\right)$.

(2) Let $G=\operatorname{Gal}(K / F)$. Let $\operatorname{tr}=\operatorname{tr}_{K / F}: K \rightarrow F$. There exists $z_{0} \in K^{\cdot}$ with $\operatorname{tr}_{*}\left\langle z_{0}\right\rangle=s_{*}\langle 1\rangle=\langle 1\rangle$. So $(-1)^{k}=\operatorname{det} \operatorname{tr}_{*}\left\langle z_{0}\right\rangle=$ $\left(\operatorname{det} \operatorname{tr}_{*}\langle 1\rangle\right) N_{K / F}\left(z_{0}\right)=N_{K / F}\left(z_{0}\right)$, as $\operatorname{tr}_{*}\langle 1\rangle=m\langle 1\rangle$. Set $z=(-1)^{k} z_{0}$. Then $N_{K / F}(z) \in F^{2}$ and $\operatorname{tr}_{*}\langle z\rangle=\left\langle(-1)^{k}\right\rangle$. Thus $\left\langle(-1)^{k}\right\rangle=\sum_{G}\langle g(z)\rangle$ and $\sum_{G}\langle 1,-g(z)\rangle=|G|\langle 1\rangle-\left\langle(-1)^{k}\right\rangle=n\langle 1\rangle \in J \subset M(K / F)$. 
(3) If $N_{K / F}(y)=1$ then we need to show $\langle 1\rangle-s_{*}\langle y\rangle \in J$. Pick $z_{0}$ and $z=(-1)^{k} z_{0}$ as in (2). Then $\langle 1\rangle-s_{*}\langle y\rangle=\langle 1\rangle-\operatorname{tr}_{*}\left\langle y z_{0}\right\rangle=\langle 1\rangle-$ $\sum_{G}\left\langle g\left(y z_{0}\right)\right\rangle=\langle 1\rangle-(-1)^{k} \sum\langle g(y z)\rangle=\langle 1\rangle+(-1)^{k} \sum\langle 1,-g(y z)\rangle-$ $(-1)^{k} m\langle 1\rangle$. As $N_{K / F}(y z)=1$ we have each $\langle 1,-g(y z)\rangle \in J$. Also $\left(1-(-1)^{k} m\right)\langle 1\rangle \in J$ by the proof of $(2)$ and so $\langle 1\rangle-s_{*}\langle y\rangle \in J$.

Corollary 1.7. Suppose $K / F$ is Galois and $s_{*}: W K \rightarrow W F$ is a ring homomorphism. Let $m=[K: F]$ and $k=(m-1) / 2$. Then $\left(m-(-1)^{k}\right)\langle 1\rangle=0$. In particular, $F$ is non-real.

Proof. Here $\left(m-(-1)^{k}\right)\langle 1\rangle \in M(K / F)=\operatorname{ker} s_{*}$, using (1.6). Yet $s_{*}\langle 1\rangle=\langle 1\rangle$, so that $\left(m-(-1)^{k}\right)\langle 1\rangle=0$.

Corollary 1.8. Suppose $K / F$ is Galois. Let $m=[K: F], k=$ $(m-1) / 2$ and $n=m-(-1)^{k}$. Let $2^{a}$ be the largest 2-power dividing $n$. If $\left|X_{K}\right|<\infty$ and the height $h(K)$ is finite then $2^{a} \in M(K / F)$.

Proof. Write $n=2^{a} \cdot b$, where $b$ is odd. If $K$ is non-real then $b\langle 1\rangle$ is a unit in $W K$ and so $2^{a} \in M(K / F)$ by (1.6)(2). Suppose then that $K$ is real. Let $Q \in X_{K}$. We Claim $U \not \subset \mathrm{pc}(Q)$, the positive cone of $Q$. Namely, suppose $U \subset \mathrm{pc}(Q)$. Then $\mathrm{pc}(Q)=U \cdot \operatorname{pc}(P)$ where $P=Q \cap F$. If $S \in X(P)-\{Q\}$ (and such an $S$ exists as $|X(P)|=$ $[K: F])$ then $\operatorname{pc}(S)=g(\operatorname{pc}(Q))$ for some $g \in \operatorname{Gal}(K / F)$. But $g(U)=U$ and $g$ fixes $F$ so that $\operatorname{pc}(S)=g(U \cdot \operatorname{pc}(P))=U \cdot \operatorname{pc}(P)=$ $\operatorname{pc}(Q)$, a contradiction.

The Claim shows that the only prime ideal to contain $M(K / F)=$ $(\{\langle 1,-y\rangle \mid y \in U\})$ is $I F$. By primary decomposition [8, 2.3], $M(K / F)$ is $I F$-primary. Since no power of $b$ is in $M(K / F) \subset I F$ we have $2^{a} \in M(K / F)$.

2. Attached primes. For modules $M$ over non-noetherian rings $R$ there are several notions of associated primes (cf. [10]). We will use three:

$$
\begin{aligned}
& \operatorname{Ass}(M)=\left\{P \in \operatorname{Spec}(R) \mid P=\operatorname{ann}_{R}(m), \text { some } m \in M\right\} \\
& \operatorname{Asf}(M)=\left\{P \in \operatorname{Spec}(R) \mid P \text { minimal over some } \operatorname{ann}_{R}(m)\right\} \\
& \operatorname{Att}(M)=\{P \in \operatorname{Spec}(R) \mid \text { for all f.g. ideals } I \subset P, \text { there } \\
&\text { exists } \left.m \in M \text { with } I \subset \operatorname{ann}_{R}(m) \subset P\right\}
\end{aligned}
$$

Ass $(M)$ is given by the usual definition of associated primes in the noetherian case. Asf $(M)$ is denoted by $\operatorname{Ass}_{f}(M)$ in [10] and $\operatorname{Att}(M)$ 
is denoted by $s K(M)$ there. Primes in $\operatorname{Att}(M)$ are called primes attached to $M$ (following Dutton [3]).

LEMMA 2.1. Let $R$ be a commutative ring and $M$ an $R$-module.

(1) $\operatorname{Ass}(M) \subset \operatorname{Asf}(M) \subset \operatorname{Att}(M)$, with equality if $R$ is noetherian.

(2) $\operatorname{Asf}(M) \neq 0$ iff $M \neq 0$.

(3) If $s, t \in \operatorname{Hom}(K, F)^{\cdot}$ then $\mathscr{A}\left(\operatorname{ker} s_{*}\right)=\mathscr{A}\left(\operatorname{ker} t_{*}\right)$ for $\mathscr{A}=$ Ass, Asf and Att.

Proof. (1) and (2) are clear cf. [10, p. 346]. For (3) note that ker $s_{*}=\langle z\rangle \operatorname{ker} t_{*}$ for some $z \in K^{\cdot}$ by $(1.1)$ and $\operatorname{ann}_{W F}(\langle z\rangle m)=$ $\operatorname{ann}_{W F}(m)$.

We remark that equality in $(2.1)(1)$ can fail at either place for nonnoetherian $R$, cf. [10].

Lemma 2.2. Let $M$ be a $W F$-submodule of $W K$. No $P(\alpha, p)$ is attached to $M$ (where $\alpha \in X_{F}, p$ an odd prime).

Proof. $\quad W K$ contains no odd dimensional zero-divisors, hence $p m \neq 0$ for all $0 \neq m \in M$. Thus if $\operatorname{ann}_{W F}(m) \subset P(\alpha, p)$ then $m \neq 0$ and $(p) \not \subset \operatorname{ann}_{W F}(m)$. So $P(\alpha, p) \notin \operatorname{Att}(M)$.

Proposition 2.3. Let $M$ be a WF-submodule of $W K$. The following are equivalent:

(1) $M \cap W_{t} K \neq 0$.

(2) $I F \in \operatorname{Att}(M)$.

(3) $I F \in \operatorname{Asf}(M)$.

(4) $z d(M)=I F$.

Proof. (1) $\rightarrow$ (2). By [3, Cor. to Prop. 6], $z d(M)=\bigcup_{P \in \operatorname{Att}(M)} P$. If $M \cap W_{t} K \neq 0$ then $2^{k} \in z d(M)$ for some $k$ and so $2^{k} \in P$, for some prime $P$ attached to $M$. But then $P=I F$.

$(2) \rightarrow(4)$. By (2.2) we have that $\operatorname{Att}(M)$ consists of some $P(\alpha)$ and possibly $I F$. Thus every $P \in \operatorname{Att}(M)$ is contained in $I F$. If $I F \in \operatorname{Att}(M)$ then $I F=\bigcup_{\operatorname{Att}(M)} P=z d(M)$.

$(4) \rightarrow(1)$ is clear as then $2 \in z d(M)$. (3) $\rightarrow$ (2) is clear by (2.1). For $(1) \rightarrow(3)$ note that we have $2^{k} m=0$ for some $m \in M . I F$ is minimal over $2^{k}\langle 1\rangle$ so that $I F \in \operatorname{Asf}(M)$.

Corollary 2.4. Let $M$ be a WF-submodule of $W K$. Then $\operatorname{Asf}(M)=\operatorname{Att}(M)$. 
Proof. We need only show $\operatorname{Att}(M) \subset \operatorname{Asf}(M)$ by (2.1). Let $P \in$ $\operatorname{Att}(M) . \quad P$ is not any $P(\alpha, p)$ by $(2.2)$ and if $P=I F$ then $P \in$ $\operatorname{Asf}(M)$ by (2.3). So suppose $P=P(\alpha)$ for some $\alpha \in X_{F}$. Then for some $m \in M \quad \operatorname{ann}_{W F}(m) \subset P(\alpha)$ and clearly $P(\alpha)$ is minimal over $\operatorname{ann}_{W F}(m)$. Thus again $P \in \operatorname{Asf}(M)$.

Theorem 2.5. Let $s \in \operatorname{Hom}(K, F)^{\bullet}$ and let $\alpha \in X_{F}$. Then $P(\alpha)$ is attached to $\operatorname{ker} s_{*}$ iff $|X(\alpha)|>1$.

Proof. Suppose first that $|X(\alpha)|>1$. Let $\beta, \gamma \in X(\alpha)$ be distinct and choose $e \in K^{*}$ with $e>_{\beta} 0$ and $e<_{\gamma} 0$. We may assume $s_{*}\langle 1\rangle=\langle 1\rangle$ by (1.1) and (2.1). Thus $x=\langle 1, e\rangle-s_{*}\langle 1, e\rangle \in \operatorname{ker} s_{*}$ and $\operatorname{sgn}_{\beta} x=2-\operatorname{sgn}_{\alpha} s_{*}\langle 1, e\rangle$ while $\operatorname{sgn}_{\gamma} x=-\operatorname{sgn}_{\alpha} s_{*}\langle 1, e\rangle$. Hence $x \notin P(\beta) \cap P(\gamma)$. We may assume $x \notin P(\beta)$.

We claim $\operatorname{ann}_{W F}(x) \subset P(\alpha)$. Suppose $r \in W F$ and $r x=0$. Then $r x \in P(\beta)$ and so $r \in P(\beta) \cap W F=P(\alpha)$. This proves the claim, and since $P(\alpha)$ is a minimal prime, shows $P(\alpha) \in \operatorname{Asf}\left(\right.$ ker $\left.S_{*}\right)=$ $\operatorname{Att}\left(\operatorname{ker} s_{*}\right)$.

Next, suppose $P(\alpha) \in \operatorname{Att}\left(\operatorname{ker} s_{*}\right)$. Assume, if possible, that $|X(\alpha)|$ $=1$. Denote by $\alpha$ also its unique extension to $K$. Suppose $\operatorname{ann}_{W F}(x)$ $\subset P(\alpha)$ for some $x \in \operatorname{ker} s_{*}$. We may assume $s=\operatorname{tr}_{K / F}$ by (2.1). Thus $0=\operatorname{sgn}_{\alpha} s_{*}(x)=\operatorname{sgn}_{\alpha} x$ by [15, III 4.5]. Hence $x \in P(\alpha)$.

Let $A=\left\{\delta \in X_{K} \mid x \in P(\delta)\right\} ; A$ is clopen. The complement $A^{\prime}$ is clopen and so is $B=\varepsilon_{K / F}\left(A^{\prime}\right)$, where $\varepsilon_{K / F}(Q)=Q \cap F$, by the Open Mapping Theorem [6, 4.9]. By the Normality Theorem [4, 3.2], there exists an $r \in W F$ such that $\operatorname{sgn}_{\delta} r=0$ if $\delta \in B$ and $\operatorname{sgn}_{\delta}(r)=2^{n}$ if $\delta \notin B$ (some fixed $n$ ). We note that $\alpha \notin B$ since $\alpha \in A, \alpha \notin A^{\prime}$ and $\varepsilon_{K / F}^{-1}(\alpha)=\{\alpha\}$ is disjoint from $A^{\prime}$.

Let $\delta \in X_{K}$. If $\delta \in A^{\prime}$ then $\beta \equiv \varepsilon_{K / F}(\delta) \in B$ and so $\operatorname{sgn}_{\delta}(r x)=0$, as $\operatorname{sgn}_{\delta}(r)=\operatorname{sgn}_{\beta}(r)=0$. If $\delta \in A$ then $\operatorname{sgn}_{\delta}(r x)=0$ as $\operatorname{sgn}_{\delta}(x)=$ 0 . Hence $r x \in W_{t} K$ and $2^{k} r x=0$ for some $k$. That is, we have $2^{k} r \in \operatorname{ann}_{W F}(x) \subset P(\alpha)$. But $\operatorname{sgn}_{\alpha}\left(2^{k} r\right)=2^{k+n}$, as $\alpha \notin B$, a contradiction.

Corollary 2.6. Suppose $\operatorname{ker} s_{*} \neq 0$. The following are equivalent:

(1) $\operatorname{ker} s_{*} \subset W_{t} K$.

(2) $M(K / F) \subset W_{t} K$.

(3) Every ordering on $F$ extends uniquely to $K$.

(4) $\operatorname{tr}_{*}\langle 1\rangle$ is a unit.

(5) $\operatorname{Att}\left(\operatorname{ker} s_{*}\right)=\{I F\}$. 
Proof. (1) $\leftrightarrow$ (2) follows as ker $s_{*}$ generates $M(K / F)$ by (1.1). (3) $\leftrightarrow(4)$ is [15, III 4.5] and [11, VIII 6.4].

$(1) \rightarrow(3)$. Let $\alpha \in X_{F}$ and let $\beta_{1}, \beta_{2} \in X(\alpha)$. Choose any $e \in K^{\cdot}$. We assume $s_{*}\langle 1\rangle=\langle 1\rangle$. Then $\langle e\rangle-s_{*}\langle e\rangle \in \operatorname{ker} s_{*} \subset W_{t} K$ and so $0=\operatorname{sgn}_{\beta_{\imath}}\langle e\rangle-\operatorname{sgn}_{\alpha} s_{*}\langle e\rangle$ for $i=1,2$. Thus $\operatorname{sgn}_{\beta_{1}} e=\operatorname{sgn}_{\beta_{2}} e$ for all $e \in K^{\cdot}$. Hence $\beta_{1}=\beta_{2}$.

$(3) \rightarrow(1)$. Let $\alpha \in X_{K}$ and set $\beta=\alpha \cap F$. Then $\operatorname{sgn}_{\beta} \operatorname{tr}_{*}(m)=$ $\operatorname{sgn}_{\alpha}(m)$ for any $m \in W K$ (tr is the trace $\left.\operatorname{tr}_{K / F}\right)$. Thus if $m \in \operatorname{ker} s_{*}$ then $\operatorname{sgn}_{\alpha} m=0$ and so $m \in W_{t} K$. Thus $\operatorname{kertr}_{*} \subset W_{t} K$ and hence $\operatorname{ker} s_{*} \subset W_{t} K$.

$(3) \rightarrow(5)$. We have $\operatorname{Att}\left(\operatorname{ker} s_{*}\right) \neq \varnothing$ by (2.1). But (2.2) and (2.5) show only IF could be attached to ker $s_{*}$. Lastly, (5) $\rightarrow(3)$ is (2.5).

For a field $E$ and form $\phi \in W E$ we write $D(\phi)$, or $D_{E}(\phi)$ if we need more precision, for the elements of $E$ represented by $\phi$. For a positive integer $m$ we will write $D(m)$ for $D(m\langle 1\rangle)$. Lastly, $D(\infty)=\bigcup_{n \geq 1} D(m)$.

Corollary 2.7. Let $s \in \operatorname{Hom}(K, F)^{\cdot}$ and suppose $s_{*}\langle 1\rangle=\langle 1\rangle$. Suppose also that $\operatorname{dim}\left(s_{*}\langle x\rangle\right)_{a n}=1$ for all $x \in K^{\cdot}$. Then:

(1) $s_{*}$ is a ring homomorphism.

(2) $m(K / F)=\operatorname{ker} s_{*}=M(K / F)=(\{\langle 1,-y\rangle \mid y \in U\})$.

(3) $U \subset D_{K}(\infty)$.

(4) Every ordering on $F$ extends uniquely to $K$.

(5) $\operatorname{Att}\left(\operatorname{ker} s_{*}\right)=\{I F\}$.

(6) For $a \in G(F), D_{K}\langle 1,-a\rangle=D_{F}\langle 1,-a\rangle\left(D_{K}\langle 1,-a\rangle \cap U\right)$.

Proof. We have $s_{*}\langle x\rangle=\left\langle N_{K / F}(x)\right\rangle$ by (1.3) and so $s_{*}$ is a ring homomorphism. Then ker $s_{*}$ is an ideal which gives (2) by (1.1) and (1.6), noting that $\langle 1\rangle-s_{*}\langle y\rangle \in \operatorname{ker} s_{*} \cap W F=0$. By (1.5) $m(K / F) \subset$ $W_{t} K$ and so if $y \in U$ then $\langle 1,-y\rangle \in W_{t} K$. Hence $U \subset D_{K}(\infty)$. Parts (4), (5) follow from (2.6) as ker $s_{*} \subset W_{t} K$.

Lastly, let $b x \in D_{K}\langle 1,-a\rangle$ where $b \in G(F)$ and $x \in U$. Then $\langle\langle-a,-b\rangle\rangle=\langle\langle-a,-x\rangle\rangle$. Apply $s_{*}$ to get

$$
\langle\langle-a,-b\rangle\rangle=s_{*}\langle\langle-a,-b\rangle\rangle=\langle\langle-a\rangle\rangle s_{*}\langle\langle-x\rangle\rangle=0 \text {. }
$$

Hence $b \in D_{K}\langle 1,-a\rangle \cap G(F)=D_{F}\langle 1,-a\rangle$. Then $x \in D\langle 1,-a\rangle$ $\cap U$. 
REMARK. (2.7) applies in the following cases:

(1) $I^{2} F=0$ (e.g. tr. d. $F=1$ ). Here we may write any $s_{*}\langle x\rangle=$ $\left\langle N_{K / F}(x)\right\rangle+\phi$ where $\phi \in I^{2} F=0$.

(2) $G(K)=\{1, a\} G(F)$. This follows from (1.4).

Corollary 2.8. If every ordering on $F$ extends uniquely to $K$ then $G(K) / G(F) \approx D_{K}(\infty) / D_{F}(\infty)$.

Proof. We may assume $W K \neq W F$. Att $(W K / W F)=\{I F\}$ by (2.6) and so $W F$ is an $I F$-primary submodule of $W K$. In particular, multiplication by $2\langle 1\rangle$ is locally nilpotent on $W K / W F$. That is, if $x \in G(K)$ then $2^{m}\langle x\rangle \in W F$ for some $m$. Hence $a x \in D_{K}\left(2^{m}\right)$ for some $a \in G(F)$. So $G(K)=G(F) D_{K}(\infty)$ and $G(K) / G(F) \approx$ $D_{K}(\infty) / D_{K}(\infty) \cap G(F)=D_{K}(\infty) / D_{F}(\infty)$.

The condition (2.3) telling when $I F$ is attached to ker $s_{*}$ is not easy to check. We give some examples. Clearly $I F \in \operatorname{Att}\left(\operatorname{ker} s_{*}\right)$ if $F$ is non-real and $W K \neq W F$. For an example with $F$ real, take $F=\mathbf{Q}$ and $K=\mathbf{Q}(\sqrt[3]{2})$. Q has a unique ordering $\alpha$ which extends uniquely, so $P(\alpha) \notin \operatorname{Att}\left(\operatorname{ker} s_{*}\right)$ by $(2.6)$. Also ker $s_{*} \neq 0$ as $\sqrt[3]{2} \notin \mathbf{Q} \cdot K^{2}$. Thus $\operatorname{Att}\left(\operatorname{ker} s_{*}\right)=\{I F\}$.

For an example with $I F \notin \operatorname{Att}\left(\operatorname{ker} s_{*}\right)$, consider the Pythagorean SAP field $K$ with automorphism $\sigma$ of odd order $n$ constructed by Ware [16]. If $F=K^{\sigma}$ then $K / F$ is Galois of degree $n$. As $|X(P)|>$ 1 for $P \in X_{F}$ we have $W K \neq W F$, while the fact that $W_{t} K=0$ implies $I F \notin \operatorname{Att}\left(\operatorname{ker} s_{*}\right)$.

In general, the property $I F \notin \operatorname{Att}\left(\operatorname{ker} s_{*}\right)$ is restrictive. We close this section by examining some of its consequences.

LEMMA 2.9. Let $[K: F]=2 k+1$ and choose $s$ such that $s_{*}\langle 1\rangle=$ $\langle 1\rangle$. Suppose IF $\notin \operatorname{Att}\left(\operatorname{ker} s_{*}\right)$. Then:

(1) $D_{K}(\infty)=D_{F}(\infty) K^{2}$.

(2) If $N_{K / F}(w) \in(-1)^{k} F^{\cdot 2}$ then $D_{F}(\infty) \subset D_{K}\langle 1,-w\rangle$.

(3) $W_{t} K=W_{t} F$.

Proof. (1) Let $w \in D_{K}(\infty)$ so that $\langle 1,-w\rangle \in W_{t} K$. Now $s_{*}\langle 1,-w\rangle \in W_{t} F$. Thus $\langle 1,-w\rangle-s_{*}\langle 1,-w\rangle \in W_{t} K \cap \operatorname{ker} s_{*}=0$ by (2.3). Then $s_{*}\langle 1,-w\rangle=\langle 1,-w\rangle, w \in F^{\cdot} K^{\cdot 2}$ and $w \in D_{F}(\infty) K^{\cdot 2}$.

(2) We have $\operatorname{det}\left(s_{*}\langle w\rangle\right)=N_{K / F}(w)=(-1)^{k}$. Then

$$
\operatorname{det}\left(\langle w\rangle-s_{*}\langle w\rangle\right)=(-1)^{k+1} w \text { and } d\left(\langle w\rangle-s_{*}\langle w\rangle\right)=w .
$$

Hence $\langle w\rangle-s_{*}\langle w\rangle=\langle 1,-w\rangle+\phi$ for some $\phi \in I^{2} K$. If $x \in D_{F}(\infty)$ 
then $\langle 1,-x\rangle\left(\langle w\rangle-s_{*}\langle w\rangle\right) \in \operatorname{ker} s_{*} \cap W_{t} K=0$. By the Arason-Pfister theorem, $\langle 1,-x\rangle\langle 1,-w\rangle=0$ and $x \in D_{K}(\langle 1,-w\rangle)$.

(3) $W_{t} K$ is generated by $\langle 1,-w\rangle, w \in D_{K}(\infty)$. Apply (1).

COROLlary 2.10. If IF $\notin \operatorname{Att}\left(\operatorname{ker} s_{*}\right)$ then $m(K / F)=0$. In particular, WK embeds into a fiber product of copies of WF. If $\left|X_{F}\right|<\infty$ then we need only finitely many copies.

Proof. If $\phi \in m(K / F), \phi \neq 0$ then $\phi \in W_{t} K$ by (1.5) and $\phi \in \operatorname{ker} s_{*}$. This contradicts (2.3). Thus $m(K / F)=0$. Write $G(K)=$ $\operatorname{gr}\{x i \mid i \in I\} \cdot G(F)$, where $\operatorname{gr}(S)$ is the group generated by $S$. Set $s_{i}(y)=\operatorname{tr}_{K / F}\left(x_{i} y\right)$ for all $y \in K$. Then $W K \rightarrow \Pi_{I} W F$ by $\phi \mapsto$ $\left(\ldots,\left(s_{i}\right)(\phi), \ldots\right)$ is injective.

Suppose $\left|X_{F}\right|<\infty$. Then $\left|X_{K}\right|<\infty$ also. Write $X_{K}=\left\{Q_{1}, \ldots\right.$, $\left.Q_{n}\right\}$. Now $\cap Q_{i}=D_{K}(\infty)=D_{F}(\infty) \dot{K}^{2}$ by (2.9). Hence

$$
[G(K): G(F)] \leq\left[\dot{K}: \bigcap Q_{i}\right] \leq 2^{n}
$$

Thus $W K$ embeds into $n$ copies of $W F$.

CoRollary 2.11. Suppose IF $\notin \operatorname{Att}\left(\operatorname{ker} s_{*}\right)$.

(1) If $\left|X_{F}\right|<\infty$ then $W K$ is a finitely generated $W F$-module.

(2) If $W F$ is noetherian then so is $W K$.

Corollary 2.12. $W F \approx W K$ iff every ordering on $F$ extends uniquely and $\operatorname{ker} s_{*} \cap W_{t} K=0$.

Proof. By (2.2), (2.3) and (2.5) we have $\operatorname{Att}\left(\operatorname{ker} s_{*}\right)=0$. Then ker $s_{*}=0$ by $(2.1)$.

REMARK. There is a partial converse to (2.8). If $W_{t} K=W_{t} F$ then IF $\notin \operatorname{Att}\left(\operatorname{ker} s_{*}\right)$. Namely, if $\phi \in W_{t} K \cap \operatorname{ker} s_{*}$ then $\phi \in W F$ and so $\phi=s_{*}(\phi)=0$. Thus $W_{t} K \cap \operatorname{ker} s_{*}=0$ and $I F \notin \operatorname{Att}\left(\operatorname{ker} s_{*}\right)$.

\section{3. $\operatorname{ker} S_{*}$ as a projective module.}

LEMMA 3.1. (1) $\operatorname{ker} s_{*}$ is projective iff $W K$ is projective.

(2) If $\operatorname{ker} s_{*}$ is free then $W K$ is free.

Proof. We may assume $s_{*}\langle 1\rangle=\langle 1\rangle$ by (1.1). Then both parts follow from $W K \approx W F \oplus \operatorname{ker} s_{*}$.

The trace of an $R$-module $M$ is:

$$
\operatorname{tr} M=\left\{\sum f_{i}\left(m_{i}\right) \mid f_{i} \in \operatorname{Hom}_{R}(M, R), m_{i} \in M\right\} .
$$


We refer to [7] for basic facts about $\operatorname{tr} M$.

Proposition 3.2. Suppose $\operatorname{ker} s_{*}$ is projective and $\operatorname{ker} s_{*} \neq 0$. Then:

(1) $\operatorname{tr}\left(\operatorname{ker} s_{*}\right)=W F$,

(2) $a n n_{W F}\left(\operatorname{ker} s_{*}\right)=0$.

Proof. (1) $\operatorname{tr}\left(\operatorname{ker} s_{*}\right)$ is an ideal so if $\operatorname{tr}\left(\operatorname{ker} s_{*}\right) \neq W F$ then $\operatorname{tr}\left(\operatorname{ker} S_{*}\right)$ is contained in a maximal ideal of $W F$. We check the two cases.

Suppose $\operatorname{tr}\left(\operatorname{ker} s_{*}\right) \subset I F$. Choose $x \in K$ such that $s_{*}\langle 1\rangle=s_{*}\langle x\rangle=$ $\langle 1\rangle$. (This is possible by (1.4) since otherwise $L(s)=\{\langle 1\rangle\}$ and $U=$ $\{\langle 1\rangle\}$. But then for any $x \in K^{\cdot}, x \in N_{K / F}(x) K^{\cdot 2} \subset F^{\cdot} K^{\cdot 2}$, as $N_{K / F}\left(x N_{K / F}(x)\right) \in K^{\cdot 2}$. This implies $W K=W F$ and ker $s_{*}=$ 0 , contrary to the assumption). Then $\langle 1,-x\rangle \in \operatorname{ker} s_{*}$. We have $I F \cdot \operatorname{ker} s_{*}=\operatorname{ker} s_{*}$ by $\left[7,3.30(\right.$ a) $]$ while $\langle 1,-x\rangle \in \operatorname{ker} s_{*} \backslash I^{2} K$ and $I F \cdot \operatorname{ker} s_{*} \subset I^{2} K$. Thus $\operatorname{tr}\left(\operatorname{ker} s_{*}\right) \not \subset I F$.

Next suppose $\operatorname{tr}\left(\operatorname{ker} s_{*}\right) \subset P(\alpha, p)$ for some $\alpha \in X_{F}$ and odd prime $p$. Let $m \geq 1$ be the largest integer with $\operatorname{tr}\left(\operatorname{ker} s_{*}\right) \subset P\left(\alpha, p^{m}\right)$; a maximum exists since $\bigcap_{m} P\left(\alpha, p^{m}\right) \subset P(\alpha) \subset I F$. Now $\operatorname{tr}\left(\operatorname{ker} s_{*}\right)=$ $\left(\operatorname{tr} \operatorname{ker} s_{*}\right)^{2}$ by [7, 3.30(a)]. Hence $\operatorname{tr}\left(\operatorname{ker} s_{*}\right) \subset P\left(\alpha, p^{m}\right)^{2} \subset P\left(\alpha, p^{2 m}\right)$, a contradiction. Thus $\operatorname{tr}\left(\operatorname{ker} s_{*}\right) \not \subset P(\alpha, p)$ and so $\operatorname{tr}\left(\operatorname{ker} s_{*}\right)=W F$.

(2) Clearly $\operatorname{tr}\left(\operatorname{ker} s_{*}\right)=W F$ is a finitely generated ideal, so $\operatorname{ann}_{W F}\left(\operatorname{ker} s_{*}\right)$ is generated by an idempotent [7, 3.30(b)]. Only 0 and 1 are idempotent in $W F\left[11\right.$, VIII 6.8] and clearly $\operatorname{ann}_{W F}\left(\operatorname{ker} s_{*}\right) \neq R$ as ker $s_{*} \neq 0$. Thus $\operatorname{ann}_{W F}\left(\operatorname{ker} s_{*}\right)=0$.

Theorem 3.3. Suppose $F$ is real and $\operatorname{ker} s_{*} \neq 0$. If some ordering on $F$ extends uniquely to $K$ then $\operatorname{ker} s_{*}$ is not projective.

Proof. $\quad$ Suppose ker $s_{*}$ is projective. Then $\operatorname{ann}_{W F}\left(\operatorname{ker} s_{*}\right)=0$ by (3.2). Let $P$ be a prime ideal attached to $W F \approx W F / \operatorname{ann}_{W F}\left(\operatorname{ker} s_{*}\right)$. Now $\left(\operatorname{ker} S_{*}\right)_{P}$ is $(W F)_{P}$-free and so:

$$
\operatorname{ann}_{(W F)_{P}}\left(\operatorname{ker} s_{*}\right)_{P}=0=\left(\operatorname{ann}_{W F}\left(\operatorname{ker} s_{*}\right)\right)(W F)_{P} .
$$

Then $P$ is attached to $\operatorname{ker} s_{*}[13$, Lemma 2]. That is, $\operatorname{Att}(W F) \subset$ $\operatorname{Att}\left(\operatorname{ker} s_{*}\right)$.

To complete the proof we need only check that every $P(\alpha), \alpha \in$ $X_{F}$, is attached to $W F$, viewed as a $W F$-module. This would yield a contradiction to (2.5). Let $\alpha \in X_{F}$ and choose $a>_{\alpha} 0$ with $a \notin F^{2}$. Then $0 \neq\langle 1,-a\rangle \in \operatorname{ann}\langle 1, a\rangle$ and ann $\langle 1, a\rangle \subset P(\alpha)$. Since $P(\alpha)$ is a minimal prime ideal we have $P(\alpha) \in \operatorname{Att}(W F)$. In the case that 
$a>_{\alpha} 0$ implies $a \in F^{\cdot 2}$ we have $X_{F}=\{\alpha\}$ and $G(F)=\{ \pm 1\}$. Thus $W F=\mathbf{Z}, P(\alpha)=\{0\}=$ ann 2 , so that again $P(\alpha) \in \operatorname{Att}(W F)$.

COROLlary 3.4. Suppose $\operatorname{ker} s_{*}$ is a non-zero projective $W F$ module. If $W_{t} F \neq 0$ then $\operatorname{ker} s_{*} \cap W_{t} K \neq 0$.

Proof. The proof of (3.3) shows $\operatorname{Att}(W F) \subset \operatorname{Att}\left(\operatorname{ker} s_{*}\right)$. If $W_{t} F \neq 0$ then $I F \in \operatorname{Att}(W F)$ by $(2.3)$ and so $I F \in \operatorname{Att}\left(\operatorname{ker} s_{*}\right)$. This implies ker $s_{*} \cap W_{t} K \neq 0$ by (2.3).

If no ordering on $F$ extends uniquely to $K$ (for example if $K / F$ is Galois) then it is possible for ker $s_{*}$ to be $W F$-projective-even for $W K$ to be $W F$-free.

Proposition 3.5. There is a real field $F$ and a Galois extension $K$ of $F$ of degree 3 such that:

(1) $W F$ and $W K$ are noetherian, and

(2) $W K$ is WF-free.

Proof. Let $\alpha=\alpha_{1}, \alpha_{2}, \alpha_{3}$ be the roots of $x^{3}-3 x+1 \in \mathbf{Q}[x]$. Note that $\mathbf{Q}(\alpha) / \mathbf{Q}$ is Galois. Let $F$ be a maximal field in $\overline{\mathbf{Q}} \cap \mathbf{R}$ not containing $\alpha(\overline{\mathbf{Q}}$ is the algebraic closure of $\mathbf{Q}) . F$ is real with the ordering induced by $\mathbf{R}$. Moreover $G(F)=\{ \pm 1\}$. Namely, if $a \in F$, $a>0$ then $F(\sqrt{a}) \subset \overline{\mathbf{Q}} \cap \mathbf{R}$ and $\alpha \notin F(\sqrt{a})$ as deg $\alpha=3$. Hence, by maximality, $F(\sqrt{a})=F$ and $a \in F^{2}$.

Let $K=F(\alpha)$. Since $x^{3}-3 x+1$ is irreducible over $F$, by construction, $K / F$ is Galois of degree 3. We claim that $K$ is Pythagorean. Suppose not. Let $\beta \in \sum K^{2}, \beta \notin K^{2}$. Note that $\beta \notin F$, as $\beta \in \sum K^{2}$ implies $\beta>0$ and so $\beta \in F$ would yield $\beta \in F^{2}$. Thus $F(\alpha)=F(\beta)=K$. Let $\sigma$ generate $\operatorname{Gal}(K / F)$ and set $\beta_{i}=\sigma^{i}(\beta)$, $i=0,1,2\left(\beta_{0}=\beta\right)$. We note that each $\beta_{i}$ is in $\sum K^{2}$. If $g(x)=$ $\operatorname{irr}(\beta, F)$ then $\left.g\left(x^{2}\right)\right)=\operatorname{irr}(\sqrt{\beta}, F)$. Thus $L=F\left(\sqrt{\beta_{0}}, \sqrt{\beta_{1}}, \sqrt{\beta_{2}}\right)$ is Galois over $F$, contains $K=F(\beta)$ and is contained in $\overline{\mathbf{Q}} \cap \mathbf{R}$.

Now $[L: F]=3 \cdot 2^{r}$ for some $r=1,2$ or 3 . Let $P$ be a Sylow 3-subgroup and let $F(Q)$ be the fixed field. Then $F(Q) \subset \overline{\mathbf{Q}} \cap \mathbf{R}$ and $\alpha \notin F(Q)$ as $\operatorname{deg} \alpha=3$ while $\operatorname{deg} Q=2^{r}$. This contradicts the maximality of $F$.

Hence $K$ is Pythagorean, and SAP since $K \subset \overline{\mathbf{Q}}$ [4, Example 1, p. 1177]. $F$ has a unique ordering so $K$ has 3 orderings. Hence $|G(K)|=8$ and $W K \approx \mathbf{Z} \sqcap \mathbf{Z} \sqcap \mathbf{Z}$ which is free over $\mathbf{Z} \approx W F$. 
The example of (3.5) yields another case where $I F \notin \operatorname{Att}\left(\operatorname{ker} s_{*}\right)$. Indeed Att $\left(\operatorname{ker} s_{*}\right)=\{P(\alpha)\}$. Also (3.5) is another example of a Pythagorean field with an automorphism of odd order (cf. [16]).

We will show that the situation of (3.5), namely, $K / F$ Galois, $W K$ noetherian and $W K W F$-free, is impossible if $F$ is non-real and non-rigid. Weaker results hold with fewer restrictions on $K$ and $F$ so we begin with no assumptions on $K$ or $F$.

LemMa 3.6. Suppose $W K$ is a free $W F$-module. Then for some index set $I$ there exists $\phi_{i} \in W K$ for $i \in I$ such that:

(1) $W K=\bigoplus_{I} W F \cdot \phi_{i}$,

(2) $\phi_{i}=\left\langle\alpha_{i}\right\rangle+\psi_{i}$ where $\alpha_{i} \in K$ and $\psi_{i} \in I^{2} K$, and

(3) $G(K)=A \times G(F)$, where $A$ is the group generated by the $\alpha_{i}$, $i \in I$.

Proof. We have $W K=\bigoplus_{I} W F \cdot \phi_{i}$ for some collection $\left\{\phi_{i} \in\right.$ $W K \mid i \in I\}$. Clearly at least one $\phi_{i}$, say $\phi_{1}$, is odd dimensional. For any even dimensional $\phi_{j}$ replace $\phi_{j}$ by $\phi_{j}-\phi_{1}$. We may thus assume (1) and (2) hold.

Now $G(K)=A \cdot G(F)$ since if $x \in G(K)$ then $\langle x\rangle=\sum r_{i} \phi_{i}$ and so $x=\operatorname{det}\langle x\rangle= \pm \prod \operatorname{det}\left(r_{i}\right) \alpha_{i} \in A \cdot G(F)$. We claim that by replacing some $\phi_{i}$ by $a \phi_{i}, a \in G(F)$, we may assume $A \cap G(F)=1$.

This is clearer if we write the $\mathrm{Z}_{2}$-vector space $G(K)$ additively. We wish to show that there exist $a_{i}(i \in I)$ in the subspace $G(F)$ such that $\operatorname{span}\left\{\alpha_{i}+a_{i} \mid i \in I\right\} \cap G(F)=\{0\}$. Choose any complementary subspace $G(F)^{\prime}$. Then every $\alpha_{i}$ has a unique expression $\alpha_{i}=a_{i}+a_{i}^{\prime}$ for some $a_{i} \in G(F)$ and $a_{i}^{\prime} \in G(F)^{\prime}$. Use these $a_{i}$.

Proposition 3.7. Suppose $F$ is non-real and $W K$ is a free $W F$ module. Then for all $f \in G(F), f \neq 1$, we have $D_{K}\langle 1,-f\rangle=$ $D_{F}\langle 1,-f\rangle$.

Proof. Write $W K=\bigoplus_{I} W F \cdot \phi_{i}$ as in (3.6). Each odd dimensional form is a unit as $F$ is non-real. Multiplication by $\phi_{1}^{-1}$ is an $W F$-module isomorphism and $\left\{\phi_{1}^{-1} \phi_{i} \mid i \in I\right\}$ satisfies (1), (2), (3) of (3.6). We may thus assume $\phi_{1}=\langle 1\rangle$. The result is clear if $W K=W F$ so we may assume $|I| \geq 2$. Write $G(K)=A \times G(F)$ as in (3.6) and let $\alpha \in A$.

Claim. $\quad W K=W F \cdot\langle 1\rangle \oplus W F \cdot\langle\alpha\rangle \oplus M$, for some $W F$-module $M$. 
We have $\langle\alpha\rangle=r_{1}\langle 1\rangle+\sum_{i \geq 2} r_{i} \phi_{i}$. If all $r_{i}(i \geq 2)$ are even dimensional then by determinants $\alpha \in G(F)$, contradicting (3.6). We may thus assume $r_{2}$ is odd dimensional. Since $F$ is non-real, $r_{2}$ is a unit in $W F$. We have:

$$
r_{2}^{-1}\langle\alpha\rangle=r_{2}^{-1} r_{1}\langle 1\rangle+\phi_{2}+\sum_{i \geq 3} r_{2}^{-1} r_{i} \phi_{i}
$$

Set $M=\bigoplus_{i \geq 3} W F \cdot \phi_{i}$. Then $\phi_{2} \in W F \cdot\langle 1\rangle+W F \cdot\langle\alpha\rangle+M$, hence $W K=W F \cdot\langle 1\rangle+W F \cdot\langle\alpha\rangle+M$. Moreover, if:

$$
s_{1}\langle 1\rangle+s_{2}\langle\alpha\rangle+m=0 \quad(m \in M)
$$

then

$$
\begin{array}{r}
s_{1}\langle 1\rangle+s_{2}\left(r_{1}\langle 1\rangle+r_{2} \phi_{2}+m^{\prime}\right)+m=0 \\
\left(s_{1}+s_{2} r_{1}\right)\langle 1\rangle+s_{2} r_{2} \phi_{2}+s_{2} m^{\prime}+m=0 .
\end{array}
$$

But $\langle 1\rangle=\phi_{1}$ and $\phi_{i}(i \geq 2)$ are independent. Thus $s_{2} r_{2}=0$. Again $r_{2}$ is a unit so $s_{2}=0$. Thus $s_{1}=0$ and $m=0$. This proves the Claim.

Now say $f \in G(F), f \neq 1$. Let $x \in D_{K}\langle 1,-f\rangle, x \notin G(F)$. Then $x=g \alpha$ for some $g \in G(F)$ and $\alpha \in A, \alpha \neq 1$. But then $\langle 1,-f\rangle\langle 1\rangle=\langle g\rangle\langle 1,-f\rangle\langle\alpha\rangle$ contradicting the Claim. Thus $D_{K}\langle 1,-f\rangle \subset G(F)$ and so $D_{K}\langle 1,-f\rangle=D_{F}\langle 1,-f\rangle$.

In the following, $B(F)$ denotes the basic part, namely those $a \in F$ with either $a= \pm 1, a$ or $-a$ not rigid (cf. [12]).

THEOREM 3.8. Suppose $F$ is non-real and $G(F)$ is finite. If ker $s_{*}$ is a finitely generated projective $W F$-module then either:

(1) $W K \approx W F[A]$ where $A=G(K) / G(F)$ or

(2) $B(F)=\{ \pm 1\}$ and $W F \approx \mathbf{Z}_{n}[C]$ with $n=2$ or 4 and $C a$ group or exponent two.

Proof. $\quad W F$ is a local ring so ker $s_{*}$, hence $W K$, is finitely generated free. Suppose $B(F) \neq\{ \pm 1\}$. Choose $f \in B(F) \backslash\{ \pm 1\}$. Set $X_{1}(K)=D_{K}\langle 1,-f\rangle$. Then $X_{1}(K)=X_{1}(F)=D_{F}\langle 1,-f\rangle$ by (3.7). For $i \geq 2$ and a field $E$ let $X_{i}(E)=\bigcup D_{E}\langle 1,-a\rangle$, over $a \in X_{i-1}(E) \backslash\{1\}$. Then by $[2,2.4]$

$$
B(K)= \pm\left(X_{1}(K) X_{2}(K)^{2} \cup-X_{1}(K) X_{3}(K)\right)=B(F) \subset G(F) .
$$

The result is then standard, see $[12,5.19]$. And if $B(F)=\{ \pm 1\}$ then $W F$ is classified as given [12, 5.21]. 
REMARK. If $W K=W F[A]$, as in (3.8)(1), then $W K$ is clearly a free $W F$-module. Suppose $B(F)=\{ \pm 1\}$ as in (3.8)(2) and $B(K) \cap$ $G(F)=\{ \pm 1\}$. We may write $G(K)=B \times C$ where $B(K) \subset B$ and $G(F)= \pm C$. Then any form in $W K$ may be written uniquely as $\sum\left\langle b_{i} c_{i}\right\rangle=\sum\left\langle c_{i 1}\right\rangle \cdot\left\langle b_{1}\right\rangle+\sum\left\langle c_{i 2}\right\rangle \cdot\left\langle b_{2}\right\rangle+\ldots$. Thus again $W K$ is a free $W F$-module. However, we know of no example of an odd degree extension $K / F$ with $W K \neq W F$ and either (3.8)(1) or (2) occurring.

We obtain a slightly weaker result if $W F$ is not noetherian.

LemMA 3.9. Let $W K=\bigoplus_{I} W F \cdot \phi_{i}$ as in (3.6). Let $\alpha, \beta \in A \backslash\{1\}$ be distinct and let $a, b, c, d \in G(F)$. If $b \alpha \in D\langle 1,-a \beta\rangle$ and $d \alpha \in$ $D\langle 1,-c \beta\rangle$ then $b=d$ and $a=c$.

Proof. We have

$$
\begin{aligned}
0 & =\langle\langle-c \beta,-d \alpha\rangle\rangle \equiv\langle\langle-a c,-d \alpha\rangle\rangle-\langle\langle-a \beta,-d \alpha\rangle\rangle \\
& \equiv\langle\langle-a c,-d \alpha\rangle\rangle-\langle\langle-a \beta,-b d\rangle\rangle\left(\bmod I^{3} K\right) .
\end{aligned}
$$

Thus $\langle\langle-a c,-d \alpha\rangle\rangle=\langle\langle-b d,-a \beta\rangle\rangle$. Apply linkage [12, 1.14]:

$$
\langle\langle-a c,-d \alpha\rangle\rangle=\langle\langle-a c,-x\rangle\rangle=\langle\langle-b d,-x\rangle\rangle=\langle\langle-b d,-a \beta\rangle\rangle
$$

for some $x \in K^{\cdot}$. Now $x \in D\langle 1,-a b c d\rangle$. If $a c \neq b d$ then $x \in$ $G(F)$ by (3.7). But $x d \alpha \in D\langle 1,-a c\rangle$ which forces $a=c$, by (3.7) again. Similarly $x a \beta \in D\langle 1,-b d\rangle$ yields $b=d$. Suppose then that $a c=b d$. Now $x d \alpha \in D\langle 1,-a c\rangle$ gives $x \in \alpha G(F)$ (unless $a=c$ and so $b=d$ ). And $x a \beta \in D\langle 1,-b d\rangle$ gives $x \in \beta G(F)$ (unless $b=d$ and so $a=c$ ). But $\alpha G(F) \cap \beta G(F)=\varnothing$. Hence $a=c$ and $b=d$.

THEOREM 3.10. Suppose $F$ is non-real and $G(F)$ is infinite. If $\operatorname{ker} s_{*}$ is a finitely generated projective $W F$-module then either:

(1) $W K \approx W F[A]$, with $A=G(K) / G(F)$ or

(2) $|B(F)|<\infty$ and $R=R_{0}[C]$ for some Witt ring $R_{0}$ and infinite group $C$ of exponent 2.

Proof. If $|B(F)|<\infty$ then $R$ is as described [12, 5.19]. Suppose $B(F)$ is infinite. Let $\alpha \in A, \alpha \neq 1$. We will show $\alpha$ is bi-rigid.

Suppose $\alpha$ is not rigid (the argument for $-\alpha$ is similar). Then $\alpha \in B(K)$ and for all $f \in B(F), f \alpha$ is not bi-rigid. Hence there exist infinitely many $f$ with $f \alpha$ not rigid (that is, if $f \alpha$ is rigid then $-f \alpha$ is not rigid). But $A$ is finite, as $W K$ is finitely generated over $W F$, so there exist distinct $f, g$ in $F$ and $\beta \in A \backslash\{1, \alpha\}$ such 
that $b \beta \in D\langle 1,-f \alpha\rangle, d \beta \in D\langle 1,-g \alpha\rangle$ for some $b, d \in F$. This contradicts (4.9).

LEMMA 3.11. If $t_{1}, \ldots, t_{n}$, and all $t_{i} t_{j}(i \neq j)$ are rigid then $D\left\langle t_{1}, \ldots, t_{n}\right\rangle=\left\{t_{1}, \ldots, t_{n}\right\}$.

Proof. By induction on $n$. Suppose $n=2$.

$$
D\left\langle t_{1}, t_{2}\right\rangle=t_{1} D\left\langle 1, t_{1} t_{2}\right\rangle=t_{1}\left\{1, t_{1} t_{2}\right\}=\left\{t_{1}, t_{2}\right\} .
$$

For $n>2$ we have by induction:

$$
D\left\langle t_{1}, \ldots, t_{n}\right\rangle=\bigcup_{i=1}^{n-1} D\left\langle t_{i}, t_{n}\right\rangle=\left\{t_{1}, \ldots, t_{n}\right\}
$$

LEMMA 3.12. Let $K / F$ be finite Galois (not necessarily of odd degree $)$. Let $t \in K \backslash F K^{2}$. Then at least one of $t, t t^{g} \quad(g \in \operatorname{Gal}(K / F))$ is not rigid.

Proof. Suppose $t$ and all $t t^{g}$ are rigid. Note $t^{g}$ is rigid as $D\langle 1, t\rangle^{g}=D\left\langle 1, t^{g}\right\rangle$. Also if $g, h \in \operatorname{Gal}(K / F)$ are distinct then $t^{g} t^{h}=g\left(t t^{h g^{-1}}\right)$ is rigid. Hence by (3.11) $D\left(\sum_{G}\left\langle t^{g}\right\rangle\right)=\left\{t^{g} \mid g \in\right.$ $\operatorname{Gal}(K / F)\}$. But $\sum\left\langle t^{g}\right\rangle=\operatorname{tr}_{*}\langle t\rangle \in W F$. Hence some $t^{g} \in G(F)$. But then $t \in G(F)$, a contradiction.

THEOREM 3.13. Let $F$ be non-real and suppose that either (i) $G(F)$ is finite and $B(F) \neq\{ \pm 1\}$ or (ii) $G(F)$ is infinite and $B(F)$ is infinite. Let $K / F$ be Galois of odd degree. Then neither $W K$ nor $\operatorname{ker} s_{*}$ are finitely generated projective WF-modules.

Proof. If $W K$ is a finitely generated projective $W F$-module then (3.8), (3.10) imply $B(K) \subset F K^{2}$ and hence if $t \in K \backslash F K^{2}$ with $K=F(t)$ then $t$ and all $t t^{g}(g \in \mathrm{Gal}(K / F))$ are bi-rigid. Namely if $t t^{g} \in F K^{2}$, say $t^{g}=a t$, then $g^{2}(t)=a(a t)=t$. Thus $t$ is fixed by $g^{2}$. As $g$ has odd order, $t$ is fixed by $g$. But then $K \neq F(t)$. This contradicts (3.12).

Ware $[16,1.6]$ shows a rigid field cannot be the Galois odd degree extension. (3.13) improves this slightly: even the case $W K \approx W F[A]$, $A=G(K) / G(F)$ cannot arise.

In a different direction we have: 
Proposition 3.14. Suppose $W K$ is a noetherian, injective $W F$ module. Then $F$ is non-real and $W F$ is Gorenstein (that is, $\mid$ ann $I F \mid$ $=2)$.

Proof. $\quad W K$ injective implies its direct summand $W F$ is injective. Thus $W F$ has injective dimension 0 and so Krull dimension 0 . Thus $F$ is non-real. Further, $W F$ is Gorenstein (cf. [1], [9]).

4. Noetherian extensions. We have given several examples of odd degree extensions $K / F$ where $W K$ is a finitely generated $W F$ module. This is necessarily the case when $X_{F}$ is finite and $I F \notin$ $\operatorname{Att}\left(\operatorname{ker} s_{*}\right)$ by (2.11). We collect here several results on the possible values of $[G(K): G(F)]$.

Proposition 4.1. Let $[K: F]=p$ be an odd prime and suppose $K / F$ is Galois. If $[G(K): G(F)]=2^{k}$ then $p \mid 2^{k}-1$.

Proof. Let $G=\operatorname{Gal}(K / F)$ and let $\sigma$ generate $G . G$ acts on $G(K) / G(F)$. Suppose $x G(F)$ is a fixed point. Then $N_{K / F}(x) \in$ $x^{p} G(F)=x G(F)$ and so $x \in G(F)$. If $x \notin G(F)$ then the orbit $\left\{\sigma^{i}(x G(F)) \mid i \in \mathbf{Z}\right\}$ has order $p$ (there is no stabilizer as $G$ is simple). Thus $p$ divides $2^{k}-1$.

EXAMPLE. Let $p$ be an odd prime and set $n=2^{p}-1$. Let $K$ be $\mathbf{Q}_{2}$ with the $n$th roots of unity adjoined. Then $K / \mathbf{Q}_{2}$ is $\mathrm{Ga}$ lois of degree $p$ [14, Prop. 16, p. 77]. By [11, p. 161] we have $\left[G(K): G\left(\mathbf{Q}_{2}\right)\right]=2^{p-1}$. This gives the minimal value of $[G(K)$ : $G(F)$ ] for $p$ such that the order of $2 \bmod p$ is $p-1$ (thus for $p=3,5,11,13,19,29,37,53,59$ etc.).

Corollary 4.2. Let $[K: F]=p_{1} p_{2} \cdots p_{t}$ with the $p_{i}$ 's prime (not necessarily distinct). Let $k_{i}$ be the least positive integer such that $p_{i} \mid 2^{k_{i}}-1$. If $K / F$ is Galois and $G(K) \neq G(F)$ then $[G(K): G(F)] \geq$ $2^{w}$, where $w=k_{1}+\cdots+k_{t}$.

Proof. We use induction on $t$. The case $t=1$ is (4.1) and if $t>1$ then choose an intermediate normal extension $L$ and apply the result to $K / L$ and $L / F$.

When $p$ is a Mersenne prime (i.e., $p=2^{k}-1$ ) then the minimal (non-trivial) square class extension for a Galois extension of degree $p$ is $p+1$. In this case we may improve (1.5). 
Proposition 4.3. Suppose $K / F$ is Galois and that $[K: F]=p$ where $p=2^{k}-1$ is a prime. If $[G(K): G(F)]=p+1$ then $m(K / F) \subset$ $\operatorname{ann}\left(2^{k}\langle 1\rangle\right)$.

Proof. Choose $s \in \operatorname{Hom}(K, F)$ with $s_{*}\langle 1\rangle=\langle 1\rangle$. There is an $x \in G(K)$ with $\operatorname{tr}_{*}\langle x\rangle=s_{*}\langle 1\rangle=\langle 1\rangle$. Now $(-1)^{p-1 / 2}=\operatorname{det} \operatorname{tr}_{*}\langle x\rangle=$ $N_{K / F}(x)$. Since $p=2^{k}-1 \quad(k \geq 2)$ we have $N_{K / F}(x)=-1$. Write $G(K)=U \times G(F)$ as in $\S 1$. There is only one (non-trivial) orbit in $G(K) / G(F)$. Thus $U=\left\{1, x_{1}, \ldots, x_{p}\right\}$ where $\sigma\left(x_{i}\right)=x_{i+1}$ (here $\sigma$ generates $\operatorname{Gal}(K / F)$ and $\left.x_{p+1} \equiv x_{1}\right)$. We may assume $x_{1}=-x$ and so $\operatorname{tr}_{*}\left\langle x_{1}\right\rangle=\langle-1\rangle$.

Let $\psi=\phi_{0}+\sum_{i=1}^{p}\left\langle x_{i}\right\rangle \phi_{i} \in m(K / F)$, where $\phi_{0}, \ldots, \phi_{p} \in W F$. Then:

$$
\begin{aligned}
& 0=\operatorname{tr}_{*} \psi=p \phi_{0}-\sum_{i=1}^{p} \phi_{i}, \\
& 0=\operatorname{tr}_{*}\left\langle x_{1}\right\rangle \psi=p \phi_{1}-\phi_{0}-\sum_{i=2}^{p} \phi_{i} .
\end{aligned}
$$

Subtraction yields $p\left(\phi_{0}-\phi_{1}\right)-\left(\phi_{1}-\phi_{0}\right)=0$ and so $2^{k}\left(\phi_{0}-\phi_{1}\right)=0$. Similarly, $2^{k}\left(\phi_{i}-\phi_{j}\right)=0$ for all $i, j$.

Now $\langle-1\rangle=\operatorname{tr}_{*}\left\langle x_{1}\right\rangle=\left\langle x_{1}, x_{2}, \ldots, x_{p}\right\rangle$. Thus $\left\langle x_{p}\right\rangle=-\left\langle 1, x_{1}, \ldots\right.$, $\left.x_{p-1}\right\rangle$. Then $\psi=\phi_{0}+\left\langle x_{1}\right\rangle \phi+\cdots+\left\langle x_{p-1}\right\rangle \phi_{p-1}-\left\langle 1, x_{1}, \ldots, x_{p-1}\right\rangle \phi_{p}$ $=\left(\phi_{0}-\phi_{p}\right)+\left\langle x_{1}\right\rangle\left(\phi_{1}-\phi_{p}\right)+\cdots+\left\langle x_{p-1}\right\rangle\left(\phi_{p-1}-\phi_{p}\right)$. Thus $2^{k} \psi$ $=0$.

(4.3) applies when $[K: F]=3$ and $[G(K): G(F)]=4$. See after (4.1) for an example of such an extension. We can improve (4.3) in this case (see the second example after (1.1)).

Corollary 4.4. Suppose $K / F$ is Galois with $[K: F]=3$ and $[G(K): G(F)]=4$. Write $U=\{1, x, y, x y\}$. Then: $0\}$.

(1) $m(K / F)=\left\{\phi_{0}\langle x\rangle+\phi_{2}\langle y\rangle \mid \phi_{i} \in W F, 4 \phi_{i}=0\right.$ and $\phi_{0}+\phi_{1}+\phi_{2}=$

(2) $m(K / F)=0$ iff $D_{F}(4) \subset D_{K}\langle 1,-x\rangle \cap D_{K}\langle 1,-y\rangle$.

(3) If $F$ is non-real and $m(K / F)=0$ then $x, y \in D_{K}(2)$.

Proof. (1) Follows from the proof of (4.3). Suppose $m(K / F)=$ 0 . If $w \in D_{F}(4)$ then for $\phi=\langle 1,-w\rangle$ we have $4 \phi=0$ and $\langle 1,-x\rangle \phi \in m(K / F)=0$. Thus $w \in D_{K}\langle 1,-x\rangle$, and similarly $w \in D_{K}\langle 1,-y\rangle$. 
If $D_{F}(4) \subset D_{K}\langle 1,-x\rangle \cap D_{K}\langle 1,-y\rangle$ and $\psi=\phi_{0}+\phi_{1}\langle x\rangle+\phi_{2}\langle y\rangle \in$ $m(K / F)$ then

$$
\begin{aligned}
\psi & =\phi_{0}+\phi_{1}\langle x\rangle-\left(\phi_{0}+\phi_{1}\right)\langle y\rangle \\
& =\phi_{0}\langle 1,-y\rangle+\langle x\rangle \phi_{1}\langle 1,-x y\rangle=0+0=0,
\end{aligned}
$$

as $\phi_{i} \in \operatorname{ann}(4)$ which is generated by $\langle 1,-w\rangle, w \in D_{F}(4)$. Thus $m(K / F)=0$, proving $(2)$.

To prove (3) note that (2) implies $D_{F}\left(2^{2+k}\right) \subset D_{K}\left(2^{k}\langle\langle-x\rangle\rangle\right)$ $\cap D_{K}\left(2^{k}\langle\langle-y\rangle\rangle\right)$. If $D_{F}(4)=G(F)$ then $-1 \in D_{K}\langle 1,-x\rangle \cap D_{K}\langle 1,-y\rangle$ and $x, y \in D(2)$. Otherwise, say $D_{F}\left(2^{k+1}\right) \neq G(F)$ and $D_{F}\left(2^{k+2}\right)=$ $G(F)$ for some $k \geq 1$. Then $-1 \in D\left(2^{k}\langle\langle-x\rangle\rangle\right)$ and $2^{k+1}\langle\langle-x\rangle\rangle=$ 0 . Thus $x \in D\left(2^{k+1}\right) \subset D\left(2^{k-1}\langle\langle-x\rangle\rangle\right)$. So $-1 \in D\left(2^{k-1}\langle\langle-x\rangle\rangle\right)$ and $2^{k}\langle\langle-x\rangle\rangle=0$. Continue until $2\langle 1,-x\rangle=0$. Similarly $2\langle 1,-y\rangle$ $=0$.

We have only a few results for non-Galois extensions.

Proposition 4.5. Let $L$ be the normal closure of $K / F$. If $L$ is real then $[K: F] \leq[G(K): G(F)]$.

Proof. Let $X_{E}(P)$ denote the set of extensions of an ordering $P$ to a field $E$. Let $Q \in X_{L}$ and set $P=Q \cap F, V=Q \cap K$. Then $\left|X_{L}(p)\right|=[L: F]$ as $L / F$ is Galois, and $\left|X_{L}(V)\right|=[L: K]$. Then $\left|X_{K}(P)\right|=[L: F] /[L: K]=[K: F]$.

Let $h(S)$ denote the number of subgroups of $G(K)$ of index 2 containing a set $S$. Let $P \in X_{F}$. Then $h(P)=|G(K) / P|-1=$ $2[G(K): G(F)]-1$. Also $h(P \cup\{-1\})=[G(K): G(F)]-1$. Thus there are $[G(K): G(F)]$ many subgroups of index 2 in $G(K)$, containing $P$ but missing -1 . These are the only possible choices for extensions of $P$ to $K$. Hence $[K: F]=\left|X_{K}(p)\right| \leq[G(K): G(F)]$.

We close with a detailed study of the smallest possible case: $[K: F]$ $=3$ and $[G(K): G(F)]=2$. We know of no such extensions.

Lemma 4.6. Suppose $[K: F]=3$ and $K / F$ is separable but not Galois. Let $L$ be the normal closure of $K$. Then:

(1) There exists a field $E$ such that $F \subset E \subset L,[L: E]=3$ and $L / E$ is Galois.

(2) $[G(K): G(F)]=\frac{[G(L): G(E)]}{\left[D_{K}\langle 1,-g\rangle: D_{F}\langle 1,-g\rangle\right]}$, for some $g \in G(F)$.

(3) $[G(K): G(F)] \leq[G(L): G(E)]$. 
Proof. We have $[L: F]=6$. Thus there exists a normal subgroup $H$ of $\mathrm{Gal}(L / F)$ of order 3. Let $E$ be the fixed field of $H$. Then $[L: E]=3$ and $E=F(\sqrt{g})$ for some $g \in G(F) \backslash\{1\}$. Suppose $K=F(e)$. Then $e \notin E$ and so $L=F(\sqrt{g})$. By [11, VII, 3.4]:

$$
\begin{aligned}
& {[G(E): G(F)]=\frac{1}{2}\left|D_{F}\langle 1,-g\rangle\right|} \\
& {[G(L): G(K)]=\frac{1}{2}\left|D_{K}\langle 1,-g\rangle\right| .}
\end{aligned}
$$

Hence the formula in (2) holds. (3) follows from (2).

Lemma 4.7. Suppose $G(K)=\{1, a\} G(F)$. Set $H=D\langle 1,-a\rangle \cap$ $G(F)$. Then for $f \in G(F)$ :

$$
\begin{aligned}
D_{K}\langle 1,-f\rangle & = \begin{cases}D_{F}\langle 1,-f\rangle & \text { if } f \notin H, \\
\{1, a\} D_{F}\langle 1,-f\rangle & \text { if } f \in H,\end{cases} \\
D_{K}\langle 1,-a f\rangle & =\{1,-a f\}\left(D_{F}\langle 1,-f\rangle \cap H\right) .
\end{aligned}
$$

Proof. By (1.4) there is an $s \in \operatorname{Hom}(K / F)$ with $s_{*}\langle 1\rangle=s_{*}\langle a\rangle=$ $\langle 1\rangle$. $(2.7)(6)$ then gives the computation of $D_{K}\langle 1,-f\rangle$. Clearly $D_{K}\langle 1,-a f\rangle=\{1,-a f\}\left(D_{K}\langle 1,-a f\rangle \cap G(F)\right)$. Then $g \in D_{K}\langle 1,-a f\rangle$ $\cap G(F)$ iff $a f \in D_{K}\langle 1,-g\rangle$ iff $g \in D_{F}\langle 1,-f\rangle$ and $g \in H$. Thus $D_{K}\langle 1,-a f\rangle=\{1,-a f\}\left(D_{F}\langle 1,-f\rangle \cap H\right)$.

Proposition 4.8. Suppose $[K: F]=3$ and $G(K)=\{1, a\} G(F)$. Then:

(1) $|D\langle 1,-a\rangle \cap G(F)| \neq 1$;

(2) If $|D\langle 1,-a\rangle \cap G(F)|=2$ then either:

(i) $\operatorname{rad}(F) \neq 1$, or

(ii) $W F$ and $W K$ are group ring extensions, or

(iii) There is a non-real Witt ring $R_{0}$ such that $W F=\mathbf{Z} \sqcap R_{0}$ and $W K=\mathbf{Z} \sqcap R_{0}[\{1, a\}]$. In particular, $\left|X_{F}\right|=\left|X_{K}\right|=1$.

Proof. (1) Suppose $|D\langle 1,-a\rangle \cap G(F)|=1$. Then (4.7) implies $a$ is bi-rigid. Thus $W K=W F[\{1, a\}]$ is a group ring extension. Let $L$ be the normal closure of $K$. Then $L=K(\sqrt{g})$ for some $g \in G(F)$. Set $E=F(\sqrt{g})$. Now $D_{K}\langle 1,-g\rangle=D_{F}\langle 1,-g\rangle$ so that $[G(K): G(F)]=[G(L): G(E)]$ by (4.6). But (4.1) implies $[G(L): G(E)] \geq 4$, a contradiction.

(2) Write $D\langle 1,-a\rangle \cap G(F)=\{1, f\}$ and suppose $\operatorname{rad}(F)=1$; in particular, $D_{F}\langle 1,-f\rangle \neq G(F)$. If $x \in G(F)-D_{F}\langle 1,-f\rangle$ then 
$D\langle 1,-a x\rangle=\{1,-a x\}$ by (4.7). Thus if there exists $g,-g \in G(F)-$ $D_{F}\langle 1,-f\rangle$ then $a g$ is bi-rigid. Now $f \in D\langle 1,-a\rangle$ so $a$ is not birigid and hence $g=a \cdot a g$ is bi-rigid. From $D_{K}\langle 1,-g\rangle=D_{F}\langle 1,-g\rangle$ we see that both $W F$ and $W K$ are group rings (with $\{1, g\}$ the group). This gives (ii).

So we may assume for all $g \in G(F)$ that either $g$ or $-g$ is in $D\langle 1,-f\rangle$. Thus $\left[G(F): D_{F}\langle 1,-f\rangle\right]=2$ and $-1 \notin D_{F}\langle 1,-f\rangle$. In particular, $D_{F}\langle 1,-f\rangle$ is an ordering on $F$. From $G(F)=\{1, f\} \times$ $D_{F}\langle 1,-f\rangle$ we get $W F=\mathbf{Z} \sqcap R_{0}$ for some Witt ring $R_{0}$.

We also have that $D_{K}\langle 1,-f\rangle=\{1, a\} D_{F}\langle 1,-f\rangle$ has index 2, in $G(K)$, and misses -1 . Thus $D_{K}\langle 1,-f\rangle$ is an ordering. Again, $G(K)=\{1, f\} \times D_{K}\langle 1,-f\rangle$. Now in $D_{K}\langle 1,-f\rangle, D\langle 1, a\rangle=\{1, a\}$ and $D\langle 1,-a f\rangle=\{1,-a f\}$. Hence $W K=\mathbf{Z} \sqcap R_{0}[\{1, a\}]$.

Lastly, (2.7) implies $\operatorname{Att}\left(\operatorname{ker} s_{*}\right)=\{I F\}$. Then (2.7) and (2.8) yield $\left|D_{K}(\infty) / D_{F}(\infty)\right|=2$. Now $D_{F}(\infty)=1 \times D_{L}(\infty)$, where $R_{0}=W L$, and $D_{K}(\infty)=1 \times D_{L}(\infty)$ unless $a \in D_{L}(\infty)$. But this only occurs if $-1 \in D_{L}(\infty)$. Hence $R_{0}$ is non-real and $\left|X_{K}\right|=\left|X_{F}\right|=1$.

\section{REFERENCES}

[1] H. Bass, On the ubiquity of Gorenstein rings, Math. Z., 82 (1963), 8-28.

[2] A. Carson and M. Marshall, Decomposition of Witt rings, Canad. J. Math., 34 (1982), 1276-1302.

[3] P. Dutton, Prime ideals attached to a module, Quart. J. Math. (2), 29 (1978), 403-413.

[4] R. Elman and T.-Y. Lam, Quadratic forms over formally real fields and pythagorean fields, Amer. J. Math., 94 (1972), 1155-1194.

[5] __ Quadratic forms under algebraic extensions, Math. Ann., 219 (1976), 21 42.

[6] R. Elman, T.-Y. Lam, and A. Wadsworth, Orderings under field extensions, J. Reine Angew. Math., 306 (1979), 7-27.

[7] C. Faith, Algebra: Rings, Modules and Categories I, Grundlehren Math. Wiss., vol. 190, Springer-Verlag, New York/Heidelberg/Berlin, 1973.

[8] R. Fitzgerald, Primary ideals in Witt rings, J. Algebra, 96 (1985), 368-385.

[9] _ Gorenstein Witt rings, Canad. J. Math., 60 (1988), 1186-1202.

[10] J. Iroz and D. Rush, Associated prime ideals in non-noetherian rings, Canad. J. Math., 36 (1984), 344-360.

[11] T.-Y. Lam, The Algebraic Theory of Quadratic Forms, Benjamin, Reading, Mass., 1980.

[12] M. Marshall, Abstract Witt rings, Queen's Papers in Pure and Appl. Math., vol. 57, Kingston, Ont., 1980.

[13] D. G. Northcott, Remarks on the theory of attached prime ideals, Quart. J. Math. (2), 33 (1982), 239-245.

[14] J.-P. Serre, Local Fields, Graduate Texts in Math., vol. 67, Springer-Verlag, New York/Heidelberg/Berlin, 1979. 
[15] W. Scharlau, Quadratic and Hermitian Forms, Grundlehren Math. Wiss., vol. 270, Springer-Verlag, Berlin/Heidelberg/New York/Tokyo, 1985.

[16] R. Ware, Automorphisms of pythagorean fields and their Witt rings, Comm. Algebra 17 (4) (1989), 945-969.

Received December 1, 1990 and in revised form July 8, 1991.

SOUTHERN ILLINOIS UNIVERSITY

CARBONDALE, IL 62901-4408 



\title{
PACIFIC JOURNAL OF MATHEMATICS
}

Founded by

\author{
E. F. BeCKeNBACH (1906-1982) F. Wolf (1904-1989)
}

\section{EDITORS}

\section{S. VARADARAJAN (Managing Editor) University of California Los Angeles, CA 90024-1555 vsv@math.ucla.edu \\ F. Michael Christ University of California Los Angeles, CA 90024-1555 christ@math.ucla.edu}

\section{Herbert Clemens}

University of Utah

Salt Lake City, UT 84112

clemens@math.utah.edu

\author{
THOMAS ENRIGHT \\ University of California, San Diego \\ La Jolla, CA 92093 \\ tenright@ucsd.edu \\ Nicholas ERcolani \\ University of Arizona \\ Tucson, AZ 85721 \\ ercolani@math.arizona.edu \\ R. FINN \\ Stanford University \\ Stanford, CA 94305 \\ finn@gauss.stanford.edu \\ VAUGHAN F. R. JONES \\ University of California \\ Berkeley, CA 94720 \\ vfr@math.berkeley.edu
}

\section{SUPPORTING INSTITUTIONS}

UNIVERSITY OF ARIZONA

UNIVERSITY OF BRITISH COLUMBIA

UNIVERSITY OF OREGON

CALIFORNIA INSTITUTE OF TECHNOLOGY

UNIVERSITY OF CALIFORNIA

UNIVERSITY OF MONTANA

UNIVERSITY OF NEVADA, RENO

NEW MEXICO STATE UNIVERSITY

OREGON STATE UNIVERSITY
SteVen KerckhofF

Stanford University

Stanford, CA 94305

spk@gauss.stanford.edu

MARTIN SCHARLEMANN

University of California

Santa Barbara, CA 93106

mgscharl@henri.ucsb.edu

HAROLD STARK

University of California, San Diego

La Jolla, CA 92093

\author{
STANFORD UNIVERSITY \\ UNIVERSITY OF HAWAII \\ UNIVERSITY OF UTAH \\ WASHINGTON STATE UNIVERSITY \\ UNIVERSITY OF WASHINGTON
}

UIV 


\section{PACIFIC JOURNAL OF MATHEMATICS}

Volume $158 \quad$ No. $1 \quad$ March 1993

Determinant identities

GEORGE W. Eyre ANDrEWS and WiLliam H. Burge

A spectral theory for solvable Lie algebras of operators

E. BOASSO and ANGEL RAFAEL LAROTONDA

Simple group actions on hyperbolic Riemann surfaces of least area

S. Allen Broughton

Duality for finite bipartite graphs (with an application to $\mathrm{II}_{1}$ factors)

MARIE CHODA

Szegő maps and highest weight representations

MARK GREGORY DAVIDSON and Ron STANKE

Optimal approximation class for multivariate Bernstein operators

ZEEV DitZIAN and XINLONG ZHOU

Witt rings under odd degree extensions

ROBERT FITZGERALD

Congruence properties of functions related to the partition function

ANTHONY D. FORBES

Bilinear operators on $L^{\infty}(G)$ of locally compact groups

Colin C. GRAham and Anthony To-Ming LAU

Nonuniqueness of the metric in Lorentzian manifolds

GEOFFREY K. MARTIN and GERARD THOMPSON

Index theory and Toeplitz algebras on one-parameter subgroups of Lie 189 groups

EFTON PARK 